\title{
Ginkgo biloba extract postconditioning reduces myocardial ischemia reperfusion injury
}

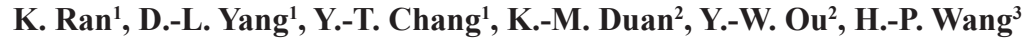 \\ and Z.-J. $\mathbf{L i}^{1}$ \\ ${ }^{1}$ Department of Anesthesiology, The Second Xiangya Hospital, \\ Central South University, Changsha \\ ${ }^{2}$ Department of Anesthesiology, The Third Xiangya Hospital, \\ Central South University, Changsha \\ ${ }^{3}$ Department of Surgery, The Second Xiangya Hospital, \\ Central South University, Changsha \\ Corresponding author: Z.-J. Li \\ E-mail: Lizhijian718@hotmail.com
}

Genet. Mol. Res. 13 (2): 2703-2708 (2014)

Received March 20, 2013

Accepted October 31, 2013

Published April 8, 2014

DOI http://dx.doi.org/10.4238/2014.April.8.14

\begin{abstract}
We examined the protective effects of Ginkgo biloba extract (EGb761) postconditioning on myocardial ischemia reperfusion injury in rabbits. Four groups of 8 white rabbits were allocated to: pseudo surgery group: the left coronary was lined without blocking for $160 \mathrm{~min}$ after thoracotomy; ischemia and reperfusion group (IR): the left anterior descending coronary artery was blocked for $40 \mathrm{~min}$ and reperfused for $120 \mathrm{~min}$; ischemic postconditioning group: the left anterior descending artery was ligated for $40 \mathrm{~min}$, reopened for $30 \mathrm{~s}$ and ligated for $30 \mathrm{~s}$, repeated three times, and then reperfused for 120 min; EGb761 postconditioning group (E): $100 \mathrm{mg} / \mathrm{kg}$ EGb761 was injected into a vein while the left coronary artery was opened for 1 min. The reperfusion took $120 \mathrm{~min}$. Internal carotid arterial blood in each group was collected for $\mathrm{cTnI}$ measurement at five times: $20 \mathrm{~min}$ before occlusion of the left coronary artery, 20 min after left coronary artery occlusion, $40 \mathrm{~min}$ after left coronary artery occlusion, $1 \mathrm{~h}$ after
\end{abstract}


myocardial reperfusion, and $2 \mathrm{~h}$ after myocardial reperfusion. Superoxide dismutase (SOD), malondialdehyde (MDA) in the centrifuged blood and myocardial infarction area were measured at the end of reperfusion. We found that the serum cTnI concentrations in the E group during reperfusion decreased significantly compared with those in the IR group. The infarction area was significantly lower in the E group than that in the IR group. The SOD activity in the E group was increased compared with that in the IR group; the MDA content decreased significantly in the E group compared with that in the IR group. We conclude that G. biloba extract postconditioning had myocardial protection effects by reducing the generation of oxygen-free radicals and increasing the antioxidant capacity of the myocardial cells.

Key words: Ginkgo biloba extract; Ischemia reperfusion injury; Myocardium

\section{INTRODUCTION}

Ginkgo biloba extract (EGb761) pretreatment can provide effective myocardial protection (Shen et al., 1998; Varga et al., 1999; Li et al., 2007). However, the ischemic time of patients cannot be predicted, and the clinical application of EGb761 pretreatment is limited. In recent years, some authors have proposed the concept of ischemic postconditioning (IPC) (Donato et al., 2007), demonstrating that it had a protective effect against the myocardial ischemia reperfusion injury in animal experiments. However, the protective effects of EGb761 postconditioning against myocardial ischemia and reperfusion have not been reported. Therefore, the aim of this study was to use EGb761 for IPC to explore the effects and the possible mechanisms of EGb761 postconditioning in the myocardial ischemia and reperfusion injury in rabbits. This study will provide the theoretical basis for the clinical application of EGb761.

Isoflurane-delayed preconditioning has a protective effect against myocardial ischemia-reperfusion injury (Shen et al., 1998; Li et al., 2007), which the exact mechanisms of the myocardial-delayed protective effect are not known. Studies have shown that myocardial ischemia-reperfusion injury is closely related to opioid receptor activation (Varga et al., 1999) and the regulation of the expression of apoptosis proteins Bcl-2 and caspase-3 (Donato et al., 2007). Therefore, the present study aimed to investigate $\mathrm{Bcl}-2$ and caspase-3 protein expression with the isoflurane-delayed pretreatment on myocardial ischemia and reperfusion in rabbits to further explore the mechanisms of the delayed myocardial protective effects.

\section{MATERIAL AND METHODS}

\section{Experimental animals and grouping}

Thirty-two healthy adult male New Zealand white rabbits were used (provided by the Experimental Animal Department of Xiangya Medical University), which weighed 2.0 to 2.5 
$\mathrm{kg}$. They were randomly divided into the following four groups, where each group consisted of 8 rabbits. 1) Pseudo-surgery group: the left coronary was lined without blocking for 160 min after thoracotomy. 2) Ischemia and reperfusion group (IR): the left anterior descending coronary artery was blocked for $40 \mathrm{~min}$ and reperfused for $120 \mathrm{~min}$. 3) Ischemic postconditioning group (IPC): the left anterior descending coronary artery was ligated for $40 \mathrm{~min}$, reopened for $30 \mathrm{~s}$ and ligated for $30 \mathrm{~s}$, repeating three times, and then reperfused for $120 \mathrm{~min}$. 4) EGb761 postconditioning group (E): $100 \mathrm{mg} / \mathrm{kg}$ EGb761 was immediately injected through a vein while the left coronary artery was opened for $1 \mathrm{~min}$, with reperfusion taking $120 \mathrm{~min}$. EGb761 (containing 24\% flavonoid glycosides and 6\% ginkgolide and bilobalide) was provided by the Shanghai Lu Yuan Industrial Company.

\section{Establishment of myocardial ischemia animal models}

The rabbit ischemia reperfusion model was established as previously described (Ran et al., 2008a,b).

\section{Cardiac troponin I (cTnI) measurement}

Blood from the internal carotid artery was collected from each group for cTnI measurement using an automatic biochemical analyzer, at five following times: 20 min before the occlusion of the left coronary artery (T1), 20 min after left coronary artery occlusion (T2), 40 min after left coronary artery occlusion (T3), $1 \mathrm{~h}$ after myocardial reperfusion (T4), and $2 \mathrm{~h}$ after myocardial reperfusion (T5).

\section{Measurements of biochemical parameters}

Carotid artery blood $(2 \mathrm{~mL})$ was collected immediately at the end of reperfusion, and the serum was separated by centrifugation at $4500 \mathrm{rpm}$ and stored at $-20^{\circ} \mathrm{C}$ for further tests. The xanthine oxidase method and the thiobarbituric salt method were applied to measure serum malondialdehyde (MDA) and superoxide dismutase (SOD) activity; the kit was purchased from Nanjing Jiancheng Bioengineering Institute, and the procedures were performed in strict accordance with the instructions.

\section{Determination of myocardial infarct size}

After $2 \mathrm{~h}$ of reperfusion, the coronary artery was blocked again, and $2 \mathrm{~mL} 2 \%$ Evans blue was injected through the internal carotid artery for myocardial staining to distinguish between ischemic (non-blue), and non-ischemic tissue (blue). The heart was quickly removed and cut into serial slices of about $2 \mathrm{~mm}$, and the thin myocardial slices were dried with filter paper. The right ventricle and the non-ischemic tissue were cut off and measured, and the ischemic myocardial slices were then placed in $0.5 \%$ TTC phosphate buffer, $\mathrm{pH} 7.4$, and incubated $\left(37^{\circ} \mathrm{C}\right)$ for $15 \mathrm{~min}$ to visualize the infarcted tissue. The qualities of necrosis (graywhite) and the non-necrotic areas (dark red) were measured. The ischemia and infarction areas and percentages of total left ventricular mass were determined and used to calculate the ischemia and infarction in the myocardial area. 


\section{Statistical analysis}

All data arereported as means \pm standard deviation, where the SPSS13.0 statistical software was used. Analysis of variance was used in the comparisons between groups, and the paired $t$-test was used in comparisons within groups. $\mathrm{P}<0.05$ was considered to be statistically significant.

\section{RESULTS}

\section{cTnI levels in different groups}

The serum cTnI concentrations in the IPC and E groups during reperfusion decreased compared with the IR group ( $\mathrm{P}<0.05)$, but there was no statistically significant difference between the IPC and E groups (Table 1).

Table 1. Plasma cTnI levels.
\begin{tabular}{llccccc}
\hline Group & $\mathrm{N}$ & $\mathrm{T}_{1}$ & $\mathrm{~T}_{2}$ & $\mathrm{~T}_{3}$ & $\mathrm{~T}_{4}$ & $\mathrm{~T}_{5}$ \\
\hline $\mathrm{S}$ & 8 & $1.23 \pm 0.55$ & $1.26 \pm 0.31$ & $1.18 \pm 0.27$ & $1.20 \pm 0.42$ & $1.33 \pm 0.14$ \\
$\mathrm{IR}$ & 8 & $1.16 \pm 0.18$ & $3.85 \pm 0.21$ & $6.34 \pm 0.38$ & $10.78 \pm 1.03$ & $13.63 \pm 2.35$ \\
$\mathrm{IPC}$ & 8 & $1.20 \pm 0.46$ & $4.13 \pm 0.47$ & $7.13 \pm 0.41$ & $8.52 \pm 1.41^{*}$ & $10.11 \pm 3.12^{*}$ \\
$\mathrm{E}$ & 8 & $1.24 \pm 0.37$ & $4.42 \pm 0.69$ & $7.83 \pm 0.55$ & $8.27 \pm 1.22^{*}$ & $9.86 \pm 2.98 *$ \\
\hline
\end{tabular}

Data are reported as means $\pm \mathrm{SD}$ in $\mathrm{ng} / \mathrm{mL} . \mathrm{S}=$ pseudo-surgery group; $\mathrm{IR}=$ ischemia and reperfusion group; IPC $=$ ischemic postconditioning group; $\mathrm{E}=\mathrm{EGb} 761$ postconditioning group. ${ }^{*} \mathrm{P}<0.05$ compared with the IR group.

\section{Myocardial infarction area in different groups}

The difference was not statistically significant between the ischemic area in the IPC and $\mathrm{E}$ groups compared with the IR group $(\mathrm{P}>0.05)$. The infarction area was significantly smaller in the IPC and E groups than in the IR group $(\mathrm{P}<0.05)$, and the IPC and $\mathrm{E}$ groups showed no statistically significant difference $(\mathrm{P}>0.05)$ (Table 2$)$.

Table 2. Myocardial infarction area and ischemic area in different groups.
\begin{tabular}{lcccccc}
\hline Group & $\mathrm{N}$ & $\begin{array}{c}\text { Left ventricular } \\
\text { myocardium }(\mathrm{g})\end{array}$ & $\begin{array}{c}\text { Ischemic } \\
\text { myocardium }(\mathrm{g})\end{array}$ & $\begin{array}{c}\text { Infarcted } \\
\text { myocardium }(\mathrm{g})\end{array}$ & $\begin{array}{c}\text { Ischemic } \\
\text { area }(\%)\end{array}$ & $\begin{array}{c}\text { Infarction } \\
\text { area }(\%)\end{array}$ \\
\hline $\mathrm{S}$ & 8 & $3.16 \pm 0.68$ & - & - & - & - \\
IR & 8 & $2.93 \pm 0.44$ & $0.88 \pm 0.35$ & $0.33 \pm 0.06$ & $33.6 \pm 3.1$ & $37.8 \pm 1.7$ \\
IPC & 8 & $3.21 \pm 0.37$ & $1.09 \pm 0.53$ & $0.27 \pm 0.08$ & $33.8 \pm 2.5$ & $24.8 \pm 2.9^{*}$ \\
E & 8 & $3.06 \pm 0.62$ & $1.11 \pm 0.67$ & $0.25 \pm 0.07$ & $34.7 \pm 3.3$ & $24.2 \pm 2.1^{*}$ \\
\hline
\end{tabular}

Data are reported as means \pm SD. $* \mathrm{P}<0.05$ compared with the IR group. For abbreviations, see legend to Table 1.

\section{MDA content and SOD activity in different groups}

At the end of reperfusion, SOD activity in the IPC and E groups increased compared with the IR group, and MDA content decreased in the IPC group and increased in the E group compared with the IR group $(\mathrm{P}<0.05)$. The difference in these two indicators was not statistically significant between the IPC and E groups (Table 3). 
Table 3. Plasma MDA content and SOD activity in different groups.

\begin{tabular}{lccc}
\hline Group & $\mathrm{N}$ & $\mathrm{MDA}(\mathrm{mM})$ & SOD $(\mathrm{kU} / \mathrm{L})$ \\
\hline $\mathrm{S}$ & 8 & $2.14 \pm 0.68$ & $158.74 \pm 8.87$ \\
IR & 8 & $9.42 \pm 0.83$ & $83.54 \pm 7.76$ \\
IPC & 8 & $6.38 \pm 0.71^{*}$ & $121.54 \pm 6.64^{*}$ \\
E & 8 & $5.87 \pm 0.90^{*}$ & $117.86 \pm 10.49^{*}$ \\
\hline
\end{tabular}

Data are reported as means \pm SD. $* \mathrm{P}<0.05$ compared with the IR group. For abbreviations, see legend to Table 1.

\section{DISCUSSION}

EGb761 is a natural active substance extracted from G. biloba, and its effects lead to improvement of blood rheology conditions and inhibition of platelet aggregation. Previous studies have found that administering EGb761 to the isolated heart or long-term to animals in vivo could significantly reduce ischemia reperfusion-induced creatine kinase release and myocardial infarction, which would provide myocardial protection (Shen et al., 1998; Varga et al., 1999; Li et al., 2007). We found that at the end of ischemia and beginning of reperfusion, instant EGb761 significantly blocked ischemia-reperfusion induced myocardial infarction, suggesting that EGb761 has postconditioning protective effects against myocardial injury.

cTnI is a regulatory protein of myocardial contractility and is composed of T, C and I subunits. The cTnI subunit structure is different from that of skeletal muscle and smooth muscle subunits, and it has a high degree of myocardial specificity which can better reflect myocardial injury (Arteaga et al., 2005). In the present study, compared with that in the only ischemia reperfusion group, the cTnI level significantly decreased in the G. biloba extract group. At the same time, the myocardial infarction area after reperfusion significantly decreased, suggesting that $G$. biloba extract treatment can significantly reduce myocardial injury, and thus, it has the similar effects as IPC. Li et at. (2007) found that nitric oxide (NO) synthase inhibitor L-NAME or myocardial sarcolemmal ATP-sensitive potassium channel (sarcKATP) blocker HMR1883 can significantly inhibit the delayed preconditioning protective effect of EGb761 against myocardial ischemia reperfusion injury. Therefore, we hypothesized that the myocardial protective effect of G. biloba extract may also be related to increased NO synthesis and the opening of the sarcKATP channel, but this needs to be further explored.

The early phase of myocardial ischemia and reperfusion shows the production of large amounts of oxygen-free radicals, which are the main factors causing ischemia and reperfusion injury, where myocardial damage is mainly expressed as lipid peroxidation. Oxygen-free radicals bind to polyunsaturated fatty acids on the myocardial cell membrane and cause lipid peroxidation and the formation of lipid peroxide (LPO). This consequently causes a series of changes in cell structure and function, ultimately leading to damage of the myocardial cells. Yang et al. (2004) showed that $1 \mathrm{~min}$ after the beginning of reperfusion, there was a release of a large amount of reactive oxygen species peaking at 4-5 min. The most important LPO among the body metabolites was MDA, so the determination of MDA could reflect the degree of lipid peroxidation, which would indirectly reflect the degree of cell damage (Liu et al., 2006). SOD is a specific enzyme of free radical scavenging in vivo; if its activity is decreased, an accumulation of oxygen-free radicals results. Thus, its activity may reflect the antioxidant function in an in vivo situation. The results of this study showed that SOD activity increased and MDA content decreased in the G. biloba extract group, suggesting that reducing the formation of oxygen-free radicals and improving the antioxidant capacity of the myocardial 
cells may be one of the important mechanisms of the postconditioning protective effects of $G$. biloba extract.

In most cases, the ischemia time showed uncertainty, where both ischemic preconditioning or pharmacological preconditioning was limited in actual clinical use. G. biloba extract preconditioning can be used after the onset of ischemia and before reperfusion to intervene in the heart muscle to reduce myocardial ischemia reperfusion injury to some degree, which is more feasible than preconditioning.

In addition, vessel wall injury, mural plaque rupture and other issues may occur with IPC, and G. biloba extract, nicorandil (Shi and Chen, 2007), sevoflurane (Chen et al., 2008), hydrogen sulfide (Ji et al., 2008) and other IPC drugs may prevent the occurrence of the above problems. This study suggests that G. biloba extract postconditioning has similar myocardial protective effects as other ischemic postconditioning methods. It will be a more convenient method for myocardial protection during myocardial IR injury, besides thrombolytic therapy and the coronary artery bypass operations.

In conclusion, this study showed that $G$. biloba extract postconditioning has myocardial protective effects by reducing the generation of oxygen-free radicals and increasing the antioxidant capacity of myocardial cells.

\section{REFERENCES}

Arteaga GM, Warren CM, Milutinovic S, Martin AF, et al. (2005). Specific enhancement of sarcomeric response to $\mathrm{Ca}^{2+}$ protects murine myocardium against ischemia-reperfusion dysfunction. Am. J. Physiol. Heart Circ. Physiol. 289: $\mathrm{H} 2183-\mathrm{H} 2192$.

Chen HT, Yang CX, Li H, Zhang CJ, et al. (2008). Cardioprotection of sevoflurane postconditioning by activating extracellular signal-regulated kinase 1/2 in isolated rat hearts. Acta Pharmacol. Sin. 29: 931-941.

Donato M, D'Annunzio V, Berg G, Gonzalez G, et al. (2007). Ischemic postconditioning reduces infarct size by activation of A1 receptors and $\mathrm{K}^{+}$(ATP) channels in both normal and hypercholesterolemic rabbits. J. Cardiovasc. Pharmacol. 49: 287-292.

Ji Y, Pang QF, Xu G, Wang L, et al. (2008). Exogenous hydrogen sulfide postconditioning protects isolated rat hearts against ischemia-reperfusion injury. Eur. J. Pharmacol. 587: 1-7.

Li NS, Zhong ZL and Jiang DJ (2007). Myocardial delayed protective effect and its mechanism of Ginkgo biloba extract, Zhong Yi Yao 38: 1046-1050.

Liu JX, Li XZ, Ma XB, Lin CR, et al. (2006). Cardio-protective effects of Corocalm on acute myocardial ischemia/ reperfusion injury in rats. Chin. J. Integr. Med. 12: 199-202.

Ran K, Duan KM, Zou DQ, Zhu R, et al. (2008a). Effects of isoflurane delayed preconditioning on nuclear factor- $\mathrm{kB}$ during myocardial ischemia reperfusion injury in rabbits. Chin. J. Emerg. Med. 17: 834-837.

Ran K, Duan KM, Zou DQ, Li ZJ, et al. (2008b). \{Effect of isoflurane delayed preconditioning on myocardial ischemia reperfusion injury in rabbits\}. Zhong Nan Da Xue Xue Bao Yi Xue Ban 33: 146-150.

Shen J, Wang J, Zhao B, Hou J, et al. (1998). Effects of EGb 761 on nitric oxide and oxygen free radicals, myocardial damage and arrhythmia in ischemia-reperfusion injury in vivo. Biochim. Biophys. Acta 1406: 228-236.

Shi B and Chen YP (2007). Nicorandil treatment of myocardial ischemia-reperfusion. Zhong Hua Ma Zui Xue Za Zhi 27: 309-312.

Varga E, Bodi A, Ferdinandy P, Droy-Lefaix MT, et al. (1999). The protective effect of EGb 761 in isolated ischemic/ reperfused rat hearts: a link between cardiac function and nitric oxide production. J. Cardiovasc. Pharmacol. 34: 711-717.

Yang XM, Proctor JB, Cui L, Krieg T, et al. (2004). Multiple, brief coronary occlusions during early reperfusion protect rabbit hearts by targeting cell signaling pathways. J. Am. Coll. Cardiol. 44: 1103-1110. 